\title{
Alterstice
}

Revue internationale de la recherche interculturelle

International Journal of Intercultural Research

Revista International de la Investigacion Intercultural

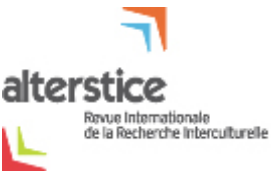

\section{In memoriam : Gustav Jahoda}

\section{Pierre Dasen et Geneviève Vermes}

Volume 6, numéro 2, 2016

URI : https://id.erudit.org/iderudit/1040628ar

DOI : https://doi.org/10.7202/1040628ar

Aller au sommaire du numéro

Éditeur(s)

Alterstice

ISSN

1923-919X (numérique)

Découvrir la revue

Citer ce document

Dasen, P. \& Vermes, G. (2016). In memoriam : Gustav Jahoda. Alterstice, 6(2),

7-11. https://doi.org/10.7202/1040628ar
Résumé de l'article

Gustav Jahoda, pionnier de la recherche interculturelle comparative et fin critique des sources de notre savoir contemporain, est décédé le 12 décembre 2016 à l'âge de 96 ans. Cet article lui rend hommage. d'utilisation que vous pouvez consulter en ligne.

https://apropos.erudit.org/fr/usagers/politique-dutilisation/ 


\section{7}

alterstice

Revue Internationale

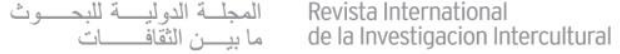

IN MEMORIAM

\section{In memoriam : Gustav Jahoda}

Pierre Dasen ${ }^{1}$ et Geneviève Vermes ${ }^{2}$

\section{Résumé}

Gustav Jahoda, pionnier de la recherche interculturelle comparative et fin critique des sources de notre savoir contemporain, est décédé le 12 décembre 2016 à l'âge de 96 ans. Cet article lui rend hommage.

\section{Rattachement des auteurs}

${ }^{1}$ Université de Genève, Suisse ; ${ }^{2}$ Université de Paris 8, France

\section{Correspondance}

pierre.dasen@unige.ch

\section{Mots clés}

comparaison ; développement ; histoire des sciences

\section{Pour citer cet article}

Dasen, P. et Vermes, G. (2016). In memoriam : Gustav Jahoda. Alterstice, 6(2), 7-12. 


\section{Introduction}

Gustav Jahoda est décédé le 12 décembre 2016 à l'âge de 96 ans. Il avait été membre honoraire de l'Association pour la recherche interculturelle (ARIC) pratiquement dès la création de celle-ci, et était un personnage important pour de nombreux membres de l'association.

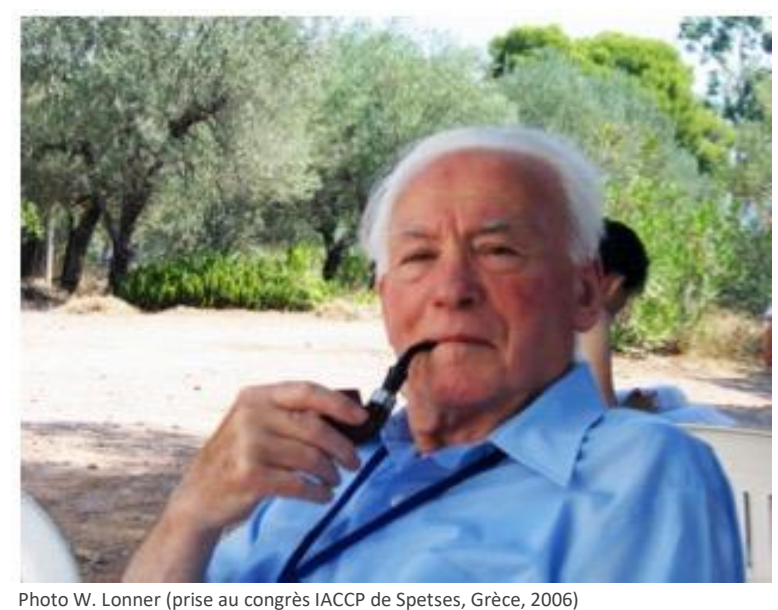

Selon le profil qu'en a donné Serpell (2013), Gustav Jahoda est né à Vienne en 1920, et a fait des études en sociologie et psychologie sociale aux universités de Vienne, Paris et Londres. II est devenu enseignant à l'Université de Glasgow (Écosse) en 1956 et, dès 1964, professeur à l'Université Strathclyde dans la même ville. II y a fondé le département de psychologie.

Gustav Jahoda a été un pionnier de la recherche interculturelle comparée. Il a effectué ses premières recherches de terrain alors qu'il occupait un poste en sociologie à l'Université de la Côte d'Or à Accra de 1952 à 1956. Par la suite, il est retourné au Ghana plusieurs fois et aussi parfois dans d'autres pays d'Afrique. Ses travaux ont porté sur différents aspects du développement cognitif et affectif, par exemple sur les liens entre les noms donnés aux enfants dans la culture Ashanti et leur personnalité (Jahoda, 1954), les erreurs de reproduction et les rotations dans le test des blocs de Kohs (Jahoda, 1978), les illusions optico-géométriques (Jahoda, 1966) et plusieurs études inspirées par la théorie de Jean Piaget (p. ex. Jahoda, 1964, 1983, 2000 ; Jahoda, Deregowski et Sinha, 1974). On ne peut que regretter que ces études soient si mal connues des chercheurs et étudiants francophones. Heureusement, en 1982, il publie un ouvrage de synthèse, Psychology and Anthropology. A psychological perspective, qui sera traduit en français en 1989.

II prend sa retraite en 1985, mais continue ses recherches en tant que professeur émérite : elles sont simplement déplacées, son « terrain » devenant la bibliothèque universitaire, les archives, internet....

Très rapidement, Gustav Jahoda a posé des questions théoriques et méthodologiques fondamentales (p. ex. Frijda et Jahoda, 1966), en particulier sur les relations entre l'anthropologie (ethnographie) et la psychologie interculturelle (Jahoda, 1982), des questions qui nous préoccupent encore de nos jours (Dasen, 2016 et à paraître). Selon lui, pour les comprendre, il était nécessaire de porter son attention sur l'histoire de ces disciplines scientifiques, et en particulier sur leurs fondements, jusque dans les écrits des $18^{\mathrm{e}}$ et $19^{\mathrm{e}}$ siècles (Jahoda, 1999a et 1999b, Jahoda et Krewer, 1997). D'abord adepte d'une conception plutôt positiviste de la psychologie, Gustav Jahoda a évolué vers une approche plus socioculturelle (p. ex. Jahoda, 1992), autrement dit d'une psychologie interculturelle comparée ("cross-cultural ») empirique vers une psychologie "culturelle » (Jahoda et Krewer, 1997). Dans la préface à un manuel (Berry, Poortinga, Segall et Dasen, 1992), il n'a pas manqué de relever que l'ouvrage était intéressant, mais qu'il l'aurait été encore plus s'il était d'avantage axé sur la psychologie culturelle ! Même après sa retraite, Gustav Jahoda n'a pas cessé de publier de nombreux livres et articles, passant des analyses historiques à une critique des courants actuels (Jahoda, 2012, 2016). 
Le travail de Jahoda était d'une extrême rigueur théorique, et sans concession. Ce travail critique majeur s'appuyait sur une connaissance érudite du corpus composé par les recherches d'aujourd'hui sur les relations entre culture et psychisme, mais pas seulement : il se donnait comme contrainte - ô combien féconde - de comprendre la construction des concepts utilisés, leur histoire. Sa qualité d'historien des savoirs et des sciences est largement reconnue. La lecture de ses publications éclaire pourquoi, comment et combien il est heuristique de nouer ainsi problèmes conceptuels, théoriques et histoire de ceux-ci. Quasiment seul à poursuivre ce travail historique en psychologie, il a en outre continué à être présent, et même actif, pour la psychologie comparative interculturelle en train de se faire. Son attention extrême à ce qui était en train de se passer, en train d'être débattu, est restée active et pointue jusqu'à ses tout derniers mois.

Il avait une grande exigence: ne rien amalgamer, ne rien réduire, ne rien gommer des problèmes liés à l'interdisciplinarité que la psychologie se propose de nouer avec les sciences tant culturelles que sociales. Ainsi se méfiait-il, avec raison, des emprunts, des transferts conceptuels non ou mal maîtrisés entre disciplines. Plus encore, il ne cessait de soumettre à examen la posture du chercheur au sein de la recherche elle-même. On peut dire qu'il a porté sur la psychologie comparative interculturelle un regard épistémologique de plus en plus poussé, dont il a fait son héritage, à notre profit.

Permettez-nous d'évoquer maintenant quelques aspects plus personnels de nos rencontres avec Gustav Jahoda.

Pierre Dasen a considéré Gustav comme son mentor depuis le jour de 1971 où ce dernier a présidé son jury de thèse.

Gustav et moi sommes restés en contact étroit, en particulier par différentes associations où nous nous retrouvions lors de congrès (ARIC, IACCP, ISSBD). Lors d'une visite chez Gustav à Glasgow en 1982, je me souviens encore de mon plaisir de découvrir que nous avions en très grande partie les mêmes livres dans nos bibliothèques. En 1986, nous éditions ensemble un numéro spécial de la revue de l'ISSBD (Dasen et Jahoda, 1986) sur la psychologie interculturelle du développement de l'enfant. Gustav parlait parfaitement le français, ce qui lui a permis de participer à plusieurs congrès de l'ARIC ainsi qu'à un colloque mémorable au Château de Cerisy-la-Salle en juin 1986 (Jahoda, 1988). Je me souviens aussi d'un congrès de l'IACCP à Pultusk, en Pologne en 2000, où les organisateurs nous avaient demandé à tous deux de figurer au programme "meet the elders " ("venez rencontrer les anciens "). J'avais été flatté d'être mis au côté de mon mentor..., mais en même temps avais pris un petit coup de vieux !

Sans I'ARIC, Geneviève Vermes n'aurait probablement pas bénéficié des échanges intellectuels de haute qualité qu'elle a pu avoir avec le regretté ami Gustav Jahoda, échanges tant de vive voix que par écrit.

Je le sollicitais : j'avais besoin d'apprendre de lui, et j'avais besoin de ses critiques toujours constructives. II se disait, modestement, avec sa grande élégance, et sa chaleureuse amitié, toujours intéressé par les hypothèses opératoires et les ouvertures théoriques que les chercheurs élaboraient pour mieux concevoir et rendre empiriquement observables les relations culture / processus psychiques / conduites individuelles, tant sous l'angle anthropologique que psychologique.

C'est dans le cadre de l'ARIC, dès ses débuts, que je le rencontre et prends connaissance de ses travaux et de la spécificité de ceux-ci, liant analyse historique et analyse théorique. Les positionnements théoriques au sein de l'ARIC donnent lieu à des débats très intenses de haute qualité. II y participe. À la fin des années 1980, je peux heureusement lui proposer la traduction en français de son manuel Psychologie et Anthropologie (1989). Celui-ci a pu ainsi constituer un noyau dur pour un enseignement reprenant le même titre et la même problématique, posant une fécondité mutuelle entre ces deux disciplines, au sein des études de psychologie (en France ces études sont organisées nationalement). Pour un temps, cet enseignement fut mis en place, non sans difficulté, celui-ci étant alors ô combien considéré comme marginal, voire iconoclaste pour le « mainstream » de la psychologie générale. Malheureusement ceci n’a 
pas encore changé aujourd'hui en France ! Cet ouvrage de Gustav Jahoda et celui de Bril et Lehalle (1988) sur Le développement psychologique est-il universel? ainsi que celui de Camilleri (1989), rédigé à la demande de l'UNESCO : Anthropologie culturelle et développement, resteront longtemps les seuls manuels disponibles en français. Ce sont encore les travaux des membres de I'IACCP qui constituent alors les références essentielles, en anglais.

Au début des années 1990, I'ARIC peut désormais, plus facilement qu'à ses débuts, publier ses travaux collectifs et ceux des chercheurs francophones œuvrant au développement de la psychologie comparative e de la psychologie interculturelle, par le biais de la collection Espaces interculturels chez l'Harmattan. En 1993, Jahoda rédige un article historique intitulé "l'Homme ou les hommes ? Lumières ou romantisme ? ", qui est publié dans cette collection (Jahoda, 1993). La lecture de cet article montre combien le travail historique sur les concepts que nous utilisons aujourd'hui dans nos recherches est indispensable. Cet article est aussi exemplaire pour qui se pose la question, comme Jahoda en 1984 : « Do we need a concept of culture?».

Jahoda poursuit ainsi l'étude de ce qu'il n'a cessé d'interroger: le rapport occidental à l'autre, la construction de cet autre, et plus spécifiquement la construction des regards savants sur l'autre, en tant que celui-ci est caractérisé par une culture autre (Jahoda, 1999a). Jahoda a, à ma demande, mené des recherches à caractère historique sur la "psychologie des peuples" de Wundt (Jahoda, 1999b). Dès la naissance de la psychologie comme savoir positif, scientifique, les questions de différences "culturelles " entre groupes (de langue, de religion, de mœurs, de contes...), sont pointées par le " grand ancêtre " psychologue Wundt, comme pertinentes pour approcher une spécificité collective d'un certain type, portée par les membres individuels (cette spécificité n'était pas encore appelée une/la " culture »). Et, selon Wundt, ces objets culturels sont psychologiquement déterminants pour l'étude des fonctions psychiques supérieures. II existerait, entre ces objets et ces fonctions, une "connivence " à mettre en évidence.

Vieille question que la psychologie traîne donc depuis son origine, et que la psychologie interculturelle d'aujourd'hui se propose de mettre au clair ! Selon Gustav Jahoda, il nous fallait, toujours plus précisément, mettre au clair « notre » usage - psychologique - de " la culture », il a encore raison.

\section{Références bibliographiques}

Berry, J., Poortinga, Y., Segall, M. et Dasen, P. (1992). Cross-cultural psychology: Research and applications. New York : Cambridge University Press.

Bril, B. et Lehalle, H. (1988). Le développement psychologique est-il universel ? Approches interculturelles. Paris : Presses universitaires de France.

Camilleri, C. (1989) Anthropologie culturelle et développement. Lausanne : Delachaux et Niestlé.

Dasen, P. (2016). La psychologie interculturelle comparée comme laboratoire quasi-expérimental. In E. Regnault et E. Costa-Fernandez (dir.). L'interculturel aujourd'hui : Perspectives et enjeux (p. 75-87). Paris : L'Harmattan, Espaces Interculturels.

Dasen, P. (à paraître). The trouble of having a psychologist sharing field work. Dans Von Poser et A. von Poser (dir.), Facets of fieldwork. Essays in honor of Jürg Wassmann. Heidelberg : Universitätsverlag Winter.

Dasen, P. et Jahoda, G. (1986). Preface. Cross-cultural human development. Special issue. International Journal of Behavioral Development, 9(4), 413-416.

Frijda, N. et Jahoda, G. (1966). On the scope and methods of cross-cultural research. International Journal of Psychology, 1, 110-127.

Jahoda, G. (1954). A note on Ashanti names in relation to personality. British Journal of Psychology, 45, 192-195. 
Jahoda, G. (1964). Children's concepts of nationality: a critical study of Piaget's stages. Child development, 35, 10811092.

Jahoda, G. (1966). Geometric illusions and environment: A study in Ghana. British Journal of Psychology, 57, 193199.

Jahoda, G. (1978). Cross-cultural study of factors influencing orientation errors in reproduction of Kohs-type figures. British Journal of Psychology, 69, 45-47.

Jahoda, G. (1982). Psychology and anthropology: A psychological perspective. London : Academic Press (traduction française : Psychologie et Anthropologie, 1989, Armand Colin).

Jahoda, G. (1983). European "lag" in the development of an economic concept: A study in Zimbabwe. British Journal of Developmental Psychology, 1, 113-120.

Jahoda, G (1984). Do we need a concept of culture? Cross-Cultural Psychology, 15,139-151.

Jahoda, G. (1988). Les études comparatives sur la perception de l'espace. In R. Bureau et D. de Saivre (dir.), Apprentissage et Cultures (p. 143-158). Paris : Karthala.

Jahoda, G. (1989). Psychologie et anthropologie. Paris : Armand Colin.

Jahoda, G. (1992). Crossroads between culture and mind. New York : Harvester/Wheatsheaf.

Jahoda, G. (1993). L'Homme ou les hommes ? Lumières ou romantisme. In F. Tanon et G. Vermes (dir.), L'individu et ses cultures (p. 62-78). Paris : L’Harmattan.

Jahoda, G. (1999a). Images of Savages. Ancien roots of modern prejudice in Western Culture. London : Routledge.

Jahoda, G. (1999b). Une esquisse de la Völkerpsychologie du Wundt. In M. Kail et G. Vermès (dir.), La psychologie des peuples et ses dérives (p. 23-32). Paris : Centre national de documentation pédagogique.

Jahoda, G. (2000). Piaget and Lévy-Bruhl. History of Psychology, 3, 218-238.

Jahoda, G. (2012). Critical reflections on some recent definitions of « culture ». Culture and Psychology, 18, 289303.

Jahoda, G. (2016). On the rise and decline of « indigenous » psychology. Culture and Psychology, 22, 169-181.

Jahoda, G., Deregowski, J. et Sinha, D. (1974). Topological and Euclidean spatial features noted by children. A crosscultural study. International Journal of Psychology, 9, 159-172.

Jahoda, G. et Krewer, B. (1997). History of cross-cultural and cultural psychology. In J. Berry, Y. Poortinga et J. Pandey (dir.), Handbook of cross-cultural psychology, second edition. Volume 1: Theory and method (p. 142). Boston : Allyn and Bacon.

Krewer, B. et Jahoda, G. (1993). Psychology and culture : Towards a solution of the Babel ? International Journal of Psychology, 28, 367-375.

Serpell, R. (2013). Jahoda, Gustav. In K. Keith (dir.), The Encyclopedia of Cross-Cultural Psychology (p. 775-776). New York : John Wiley. 